\title{
THE USAGE OF ADAPTIVE ASSESSMENTS IN THE COMPUTER SCIENCE LEARNING PROCESS: A CASE STUDY FOR JAVA
}

\author{
Bogdan IANCU \\ The Bucharest University of Economic Studies, Romania \\ bogdan.iancu@ie.ase.ro
}

\begin{abstract}
Adaptive assessments are becoming more and more popular as they are used on a large scale for evaluating programming knowledge by industry leaders such as Pluralsight. In this paper we propose a simple decision tree-based adaptive model for evaluating students' knowledge related to Java programming. The model is making use of a lower number of questions of different difficulties compared to a classical approach. A discrete model was preferred to a more complex Item Response Theory statistical one (such as 1PL or Rasch) due to its simplicity and to the fact that it can be implement with the usage of a classical test theory system. The adaptive assessment model was evaluated on a sample of 82 students from the bachelor's degree program. The results obtained by the proposed model are promising, especially when it comes to labeling students' performance on the assessment by a binary classification technique in the form of passed or failed.
\end{abstract}

Keywords: CAT, CAA, IRT, e-learning

JEL classification: C61, C63, C88

DOI: $10.24818 / \mathrm{ie} 2020.01 .02$

\section{Introduction}

Adaptive assessments are a special category of tests that adjust their content to the examinee's ability level. When a computer algorithm is used in order to determine the next optimal item, they are usually called Computerized Adaptive Tests (CAT). The most common methodology for implementing CATs comes from the domain of psychometrics and it is called Item Response Theory (IRT).

Classical Test Theory (CTT) is used on a large scale when it comes to evaluate students' performance in the Romanian higher education. However, IRT is considered a better approach, being used for a long time at different levels within the national education systems from other countries [1]. While classical tests are usually unidimensional (they measure a single ability by using only one scale), the adaptive ones can also help in obtaining a detailed picture of an examinee's strengths and weaknesses [2].

In the case of CTT, all the items (e.g. questions from a test) are considered equal and the final score is computed by summing the individual scores of all the items. In an IRT systems, items are scored differently depending on their difficulty. For example, if two examinees score 6 out of 10 in a classical test with 10 questions, both will have the final score $60 \%$. On the other hand, if two examinees score 6 out of 10 in a test that uses IRT, their final scores might be different depending on the difficulty level and the order of the questions that they answered correct or incorrect.

They are multiple ways of measuring the final scores in an IRT system, as emphasized in the next chapters. Nevertheless, our main objective in this paper was to determine if adaptive assessments are a viable approach for testing students who learn Java programming (one ability - Java knowledge, according to the IRT theory), rather than building an IRT system per se. 
www.conferenceie.ase.ro

\section{Short literature review}

IRT is used nowadays in the context of Computer Based Testing (CBT), especially CAT, a category of adaptive tests generated with the help of a computer algorithm that can evaluate one's ability in a certain domain [3]. Usually CATs or CAAs (Computer Adaptive Assessments) are part of a larger adaptive e-learning system [4], [5].

Paper [6] provides an extensive survey of existing automated assessment approaches related to the programming domain. Several features are identified and divided into two categories: dynamic and static. Dynamic features include functionality, efficiency, testing skills, and special features, while static ones include coding style, programming errors, software metrics, design, and special features. Usually, adaptive assessments make use of item banks that are manually or automatically annotated with certain difficulty levels [7].

Also related to evaluating programming abilities, papers [8], [9] and [10] present different approaches for evaluating students who learn a new programming language. Paper [8] describes the creation of a Programming Adaptive Testing (PAT) system, a web based IRT application that uses an item bank of questions with difficulties adapted to Bloom's taxonomy. Article [9] presents an adaptive e-learning system used by students who learn the Java programming language (called QuizJET). The system is making use of parameterized questions instead of static ones. Paper [10] proposes a methodology based on IRT for evaluating students' knowledge of object-oriented programming.

Similar approaches are taken by the industry as well. Pluralsight representatives had announced via a white paper that they are using IRT to compute the value of the Skill IQ, a quotient that can tell one's proficiency in a certain technology [11].

Even tough different approaches are used to implement adaptive assessments with the usage of IRT, like Bayesian-based methods or Neural Networks-based methods [10], in this paper we chose a different approach inspired by the methodology proposed in [3]. The model is based on a decision tree and it is described in greater details in the next chapter. We chose this approach due to the possibility of adjusting an existing CTT system to an adaptive model. This wouldn't have been possible if a Bayesian model-based system was used, and the financial investment in a new system that has a built-in IRT engine isn't possible yet.

\section{Proposed adaptive assessments model}

The proposed adaptive assessment model uses a decision tree across 10 questions divided into 4 difficulty levels (easy, medium-low, medium-high and hard) and evaluates each student with a grade from 1 (lowest) to 5 (highest). The item bank is composed of questions previously used in gamified quizzes (from the Kahoot platform) with the ex-students that learned the same curriculum as the examined ones and is presented in extenso in [12]. The individual item difficulty was computed based on the previous answers of ex-students by using quartiles in the following way: Q1 - easy (questions answered correct by more than $75 \%$ percent of the students), Q2 - medium-low (questions answered correct by more than $50 \%$ of the students, but less than 75\%), Q3 - medium-high (more than $25 \%$ of students provided correct answers, but less than 50\%) and Q4 - hard (less than 25\% of students answered these questions correctly). In this way we wanted to test also if the item bank can use a feedback loop in order to reevaluate the difficulty of the questions based on previous answers [11]. The questions have a single correct answer and include only theory and source code evaluation, also similarly to the question bank used in [11]. A graphical representation of our model, inspired by the model used in [3] is presented in Figure 1. Instead of using a statistical function (such as the Rasch model for example [13]) that would have given us a floating-point number as result, we preferred a discrete approach in which the result is an integer. The model can be scaled and allows an exponential reduction of the needed questions, compared to a classical approach. If 
the number of questions needed by our model in order to compute the final score is $n$, then the number of questions needed by a classical approach would be $\frac{n(n+1)}{2}$. For the model presented below, the number of questions needed in order to compute the final score is $4(n=4)$, instead of 10 questions needed by a classical one.

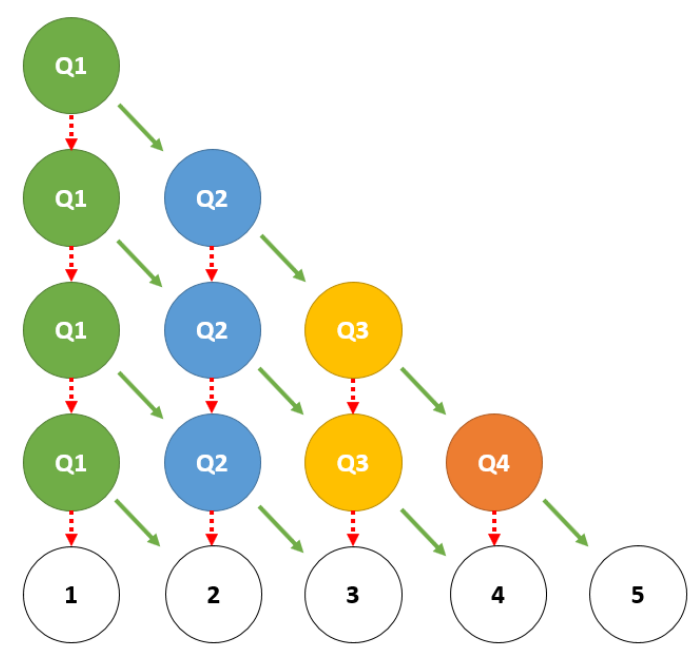

Figure 1. Graphical representation of the model (based on [3]). Q1-Q4 represent the difficulty levels, 1-5 the final grade of the student, red dotted arrows incorrect answers and green solid arrows correct answers

The main objective was to determine if an adaptive assessment can produce better results than a classical approach for the same item bank. In order to evaluate this, a classical test was conducted on 82 students from the Java programming class. The test included all the 10 questions ordered by difficulty. The students were announced that the test is just a selfevaluation one and that the final grade will not be taken into consideration. Also, at the end of the test, they were asked to self-evaluate their score on a scale from 1 to 5 .

After the results were gathered, an adaptive test simulation was conducted on every student's data with the usage of the proposed model. Table 1 shows a snippet of the aggregated data.

Table 1. A snippet of the aggregated data for the developed test (columns B, C and D are normalized and display values from 1 to 5)

\begin{tabular}{|c|c|c|c|}
\hline A. Participant ID & $\begin{array}{c}\text { B. Total points (classical } \\
\text { approach) }\end{array}$ & $\begin{array}{c}\text { C. Estimated points } \\
\text { (adaptive model) }\end{array}$ & $\begin{array}{c}\text { D. Participant self- } \\
\text { evaluation }\end{array}$ \\
\hline 1 & 4 & 3 & 5 \\
\hline 2 & 4 & 4 & 4 \\
\hline 3 & 4 & 5 & 3 \\
\hline 4 & 3 & 4 & 3 \\
\hline 5 & 3 & 3 & 1 \\
\hline 6 & 3 & 3 & 3 \\
\hline 7 & 3 & 3 & 4 \\
\hline 8 & 2 & 3 & 4 \\
\hline 9 & 3 & 4 & 3 \\
\hline 10 & 2 & 3 & $\ldots$ \\
\hline$\ldots$ & $\ldots$ & $\ldots$ & \\
\hline
\end{tabular}


www.conferenceie.ase.ro

\section{Results and discussions}

We started by analyzing the descriptive statistics for our data. All the three data series had the same median (equal to 3), mode (also 3) and the mean was similar (3.28 for column B from the table above, 3.39 for column $\mathrm{C}$ and 3.29 for column $\mathrm{D})$. The standard error was 0.09 for $\mathrm{B}, 0.1$ for $\mathrm{C}$ and 0.09 for $\mathrm{D}$, so similar as well. The distribution of the values from the data series can be observed in the Figure 2 below.

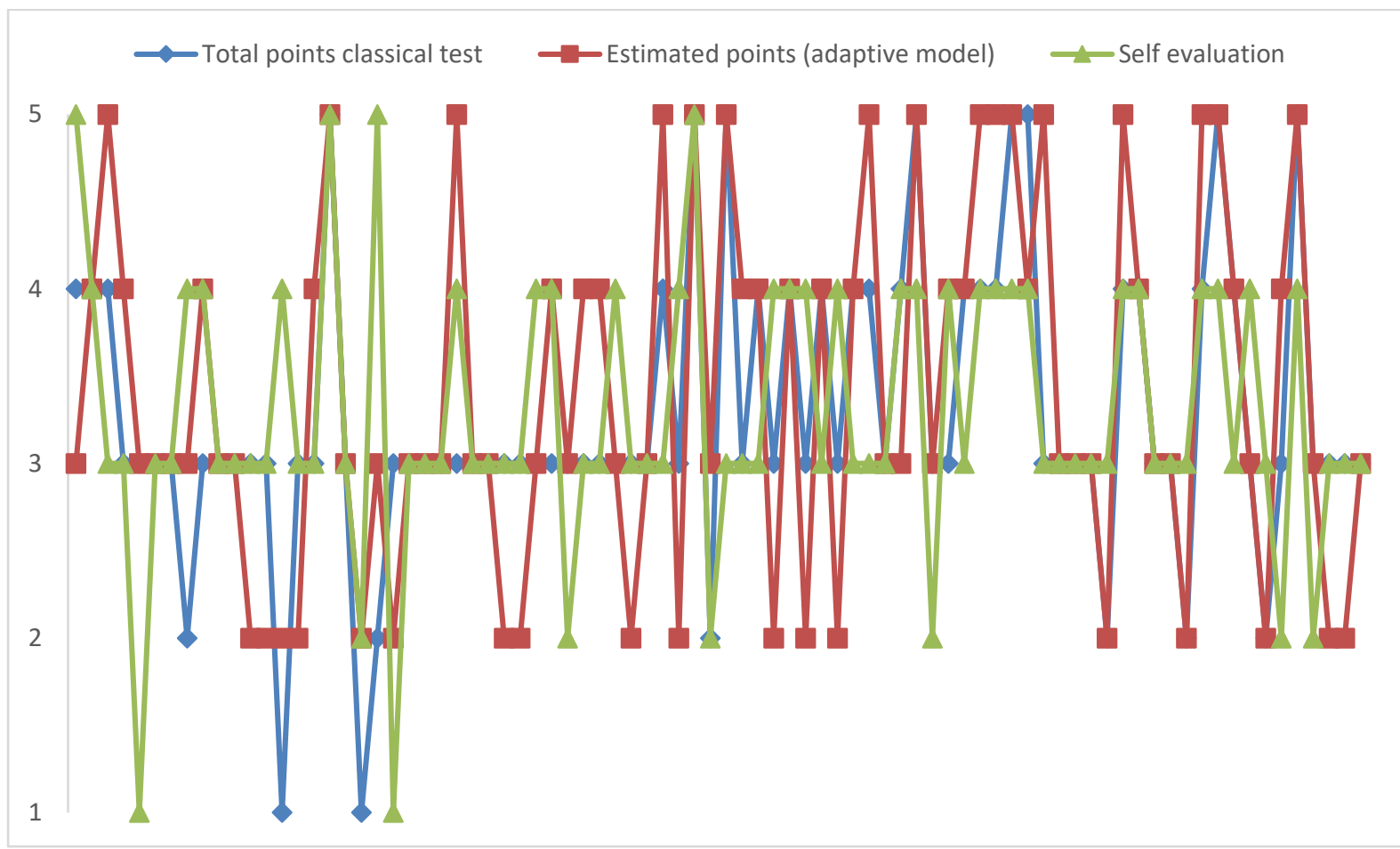

Figure 2. A chart representing the distribution of the three data series ( $B, C$ and $D$ from Table 1$)$

Based on the graphical representation of the values, we tested the correlation between the data series. The correlation coefficient between the grades obtained at the full test and the grades obtain by applying the adaptive model with just 4 questions was 0.71 . Also, the correlation between the grades obtained by the adaptive model and the self-evaluated grades was 0.31 , while the one between the grades obtained at the full test and the self-evaluation was 0.36 .

The results obtained by our model are encouraging having in consideration that they are similar to the ones obtained by the classical one, but it needs just 4 questions instead of 10 . However, we must reckon that the classical model seems to be slightly better at evaluating students, at least if we compare this with their self-evaluation. Also, we can observe from Figure 2, that the proposed adaptive model has a tendency to overestimate students with very low scores.

We should emphasize also the fact that the model was used to test students who learn Java programming and might produce different results for a different item bank. Nevertheless, if we would consider the students with grades above or equal to 3 as passed and the other ones as failed, the overestimation of the model for the low scores would not matter anymore. We have also tested the performance of our model for a binary classification decision (passed or failed) by using the $\mathrm{F}_{1}$ score (the harmonic mean between precision and recall). The obtained value for the $F_{1}$ score was 0.82 which suggests that the model has a high accuracy of determining the final decision.

Other advantages of the proposed adaptive model by comparison with the classical approach are given by the fact that it is using a low number of questions from the item bank and that each 
www.conferenceie.ase.ro

student will have its own navigation path through questions. This can discourage plagiarism and the test takers that want just to see as many items from the item bank as possible.

\section{Conclusions and future work}

In this paper we wanted to test if the CATs can be an alternative to CTT for evaluating students' knowledge related to Java programming. Instead of using a classical IRT approach like the 1PL or the Rasch model, just to name a few, we chose a simpler one based on a discrete decision three that allowed us to adapt a CTT tool to the new paradigm [3]. The content of the item bank and the way the items were auto evaluated in terms of difficulties were inspired by the industry approach used at Pluralsight [11].

The results are promising and can help us to dramatically reduce the number of questions needed in order to evaluate a student. The model scored a high accuracy when it comes to predict examinee's final decision related to the test (passed or failed).

Future work includes the usage of the open source algorithm developed by Pluralsight and available at [14], in order to develop a custom IRT system per se. The new system will overcome the current system in terms of complexity and accuracy. Also, we take into consideration to do a simulation for a larger item bank and to compare the obtained result with the current ones.

\section{Acknowledgment}

This paper was co-financed from the Human Capital Operational Program 2014-2020, project number POCU / 380/6/13/125245 no. 36482 / 23.05.2019 "Excellence in interdisciplinary PhD and post-PhD research, career alternatives through entrepreneurial initiative (EXCIA)", coordinator The Bucharest University of Economic Studies.

I would like to thank my students that agreed to help me with fulfilling the Java test although it was anonymous, and no extra points were given.

\section{References}

[1] R. McKinley and N. Kingston, "Exploring the use of IRT equating for the GRE Subject Test in Mathematics," ETS Research Report Series, vol. 1, pp. i-35, 1987.

[2] B. Heeren, J. Jeuring, S. Sosnovsky, P. Drijvers, P. Boon, S. Tacoma and J. Koop, "FineGrained Cognitive Assessment Based on Free-Form Input for Math Story Problems," in European Conference on Technology Enhanced Learning, Springer, Cham, 2018.

[3] P. Molins-Ruano, C. González-Sacristán, F. Díez, P. Rodriguez and G. M. Sacha, "Adaptive Model for Computer-Assisted Assessment in Programming Skills," International Journal of Engineering Education, vol. 31, no. 3, 2014.

[4] N. El Faddouli, B. E. Falaki, M. K. Idrissi and S. Bennan, "Towards an adaptive competency-based learning system using assessment," International Journal of Computer Science Issues (IJCSI), vol. 8, no. 1, p. 265, 2011.

[5] A. Zamfiroiu, "Security Management for Mobile Learning Systems," in Conference proceedings of» eLearning and Software for Education «(eLSE), 2018.

[6] K. M. Ala-Mutka, "A survey of automated assessment approaches for programming assignments," Computer science education, vol. 15, no. 2, pp. 83-102, 2005.

[7] D. Cella, R. Gershon, J.-S. Lai and S. Cho, "The future of outcomes measurement: item banking, tailored short-forms, and computerized adaptive assessment," Quality of Life Research, vol. 16, no. 1, pp. 133-141, 2007. 
www.conferenceie.ase.ro

[8] D. I. Chatzopoulou and A. A. Economides., "Adaptive assessment of student's knowledge in programming courses," Journal of Computer Assisted Learning, vol. 26, no. 4, pp. 258269, 2010.

[9] I. Hsiao, S. Sosnovsky and P. Brusilovsky, "Guiding students to the right questions: adaptive navigation support in an E-Learning system for Java programming," Journal of Computer Assisted Learning, vol. 26, no. 4, pp. 270-283, 2010.

[10] Y. L. P. Vega, J. C. G. Bolaños, G. M. F. Nieto and S. M. Baldiris, "Application of item response theory (IRT) for the generation of adaptive assessments in an introductory course on object-oriented programming," in 2012 Frontiers in Education Conference Proceedings, IEEE, 2012.

[11] Pluralsight, "Accuracy and Pluralsight's adaptive assessment engine," 2019. [Online]. Available:

https://www.pluralsight.com/content/dam/pluralsight2/product/iris/AdaptiveAssessments_ af_v1.pdf. [Accessed April 2020].

[12] B. Iancu, "Gamification Applied in Computer Science Education: A Preliminary Approach," Economy Informatics, vol. 19, no. 1, pp. 52-58, 2019.

[13] T. Bond, Applying the Rasch model: Fundamental measurement in the human sciences, Routledge, 2015.

[14] Pluralsight, "GitHub Repo," October 2018. [Online]. Available: https://github.com/pluralsight/irt_parameter_estimation/. [Accessed April 2020]. 